\title{
Extubation process in bed-ridden elderly intensive care patients receiving inspiratory muscle training: a randomized clinical trial
}

This article was published in the following Dove Press journal:

Clinical Interventions in Aging

19 October 2012

Number of times this article has been viewed

\author{
Samária Ali Cader ${ }^{\prime}$ \\ Rodrigo Gomes de Souza \\ Vale' \\ Victor Emmanuel Zamora ${ }^{2}$ \\ Claudia Henrique Costa ${ }^{2}$ \\ Estélio Henrique Martin \\ Dantas' \\ 'Laboratory of Human Kinetics \\ Bioscience, Federal University of \\ Rio de Janeiro State, ${ }^{2}$ Pedro Ernesto \\ University Hospital, School of \\ Medicine, State University of Rio de \\ Janeiro, Rio de Janeiro, Brazil
}

Background: The purpose of this study was to evaluate the extubation process in bed-ridden elderly intensive care patients receiving inspiratory muscle training (IMT) and identify predictors of successful weaning.

Methods: Twenty-eight elderly intubated patients in an intensive care unit were randomly assigned to an experimental group $(n=14)$ that received conventional physiotherapy plus IMT with a Threshold IMT $^{\circledR}$ device or to a control group $(n=14)$ that received only conventional physiotherapy. The experimental protocol for muscle training consisted of an initial load of $30 \%$ maximum inspiratory pressure, which was increased by $10 \%$ daily. The training was administered for 5 minutes, twice daily, 7 days a week, with supplemental oxygen from the beginning of weaning until extubation. Successful extubation was defined by the ventilation time measurement with noninvasive positive pressure. A vacuum manometer was used for measurement of maximum inspiratory pressure, and the patients' Tobin index values were measured using a ventilometer.

Results: The maximum inspiratory pressure increased significantly (by $7 \mathrm{~cm} \mathrm{H}_{2} \mathrm{O}, 95 \%$ confidence interval [CI] 4-10), and the Tobin index decreased significantly (by 16 breaths/ $\mathrm{min} / \mathrm{L}, 95 \% \mathrm{CI}-26$ to 6 ) in the experimental group compared with the control group. The Chi-squared distribution did not indicate a significant difference in weaning success between the groups $\left(\chi^{2}=1.47 ; P=0.20\right)$. However, a comparison of noninvasive positive pressure time dependence indicated a significantly lower value for the experimental group $(P=0.0001 ; 95 \%$ CI 13.08-18.06). The receiver-operating characteristic curve showed an area beneath the curve of $0.877 \pm 0.06$ for the Tobin index and $0.845 \pm 0.07$ for maximum inspiratory pressure.

Conclusion: The IMT intervention significantly increased maximum inspiratory pressure and significantly reduced the Tobin index; both measures are considered to be good extubation indices. IMT was associated with a reduction in noninvasive positive pressure time in the experimental group.

Keywords: inspiratory muscle training, rehabilitation, extubation

\section{Introduction}

Maximum respiratory system function is reached between the ages of approximately 20 and 25 years. As people age, there is a gradual reduction in pulmonary performance, and in elderly people these physiological alterations can seriously impact cardiorespiratory functional reserve. ${ }^{1}$ In addition, respiratory muscle strength is also affected by poor nutrition and sarcopenia, both of which are associated with aging and bedridden patients. ${ }^{2-4}$

In $20 \%-25 \%$ of cases, patients have difficulty in discontinuing the use of mechanical ventilation. ${ }^{5}$ Weaning begins with the reduction of ventilatory support, at which
Correspondence: Samária Ali Cader Rua Jorge Emílio Fontenelle, 550 Bloco 2a Apto 202 Rio de Janeiro, Brasil CEP 22790-I40 Tel +55 2l 31509501

Email samariacader@gmail.com 
point the patients become capable of some independent ventilation, and ends with removal of the patient from mechanical ventilation, including extubation (removal of the endotracheal tube). ${ }^{6,7}$

Numerous indices have been utilized to predict the success of interruption of ventilatory and extubation following mechanical ventilation. These different indices reflect the mechanics of respiration, ie, pulmonary gas exchange, respiratory control, and functioning of the respiratory musculature. ${ }^{8}$ Although there are a variety of predictors, Vallverdú et $\mathrm{al}^{9}$ observed that the Tobin index and maximum inspiratory pressure are good predictors of weaning success. A common finding in patients who fail weaning and extubation is a raised Tobin index (respiratory frequency/ vital capacity, in liters)..$^{10}$ Currently, maximum inspiratory pressure is used to evaluate the strength of the inspiratory musculature. ${ }^{11}$

Chronic dependence on mechanical ventilation is not only a major medical problem, ${ }^{2}$ but also produces extreme discomfort for patients, with important physiological complications and negative social implications and restrictions. ${ }^{12}$ Therefore, some investigators have suggested use of inspiratory muscle training (IMT) to reduce respiratory muscle fatigue ${ }^{13}$ and facilitate weaning from mechanical ventilation. ${ }^{14}$ However, these studies have only involved tracheostomized patients. This study aimed to evaluate the extubation process in bedridden elderly patients receiving IMT and correlate successful predictors of weaning.

\section{Materials and methods Sample}

A randomized trial was conducted with participants from the intensive care unit of a single hospital in Brazil. After confirming their eligibility and baseline measurements, the participants were randomly allocated to either an experimental group or a control group. The enrolling investigator contacted another investigator to request participant allocation from a concealed list of random allocations that had been generated by drawing numbers from a bag. This other investigator was not otherwise involved in the study.

During the recruitment period, 198 patients were screened, and 67 were eligible and monitored daily to assess their readiness to begin weaning. Of these 67 patients, 20 were tracheostomized, five died, and one was transferred to another center before weaning began. The remaining 41 were randomized, with 21 assigned to the experimental group and 20 to the control group. However, four participants in each group died before extubation. Three participants in the experimental group and two in the control group were tracheostomized before extubation. The experimental group ( $n=14$, age $82 \pm 4$ years, mechanical ventilation period 10 days) underwent conventional physiotherapy plus IMT with a Threshold IMT $^{\circledR}$ device (Philips Respironics, Murrysville, PA), and the control group ( $\mathrm{n}=14$, age $81 \pm 6$ years, mechanical ventilation period 11 days) received only conventional physiotherapy that favored inspiratory muscle strengthening.

To be included, the sample participants were required to have undergone mechanical ventilation for at least 48 hours in a controlled manner, ${ }^{13}$ because intubation is diagnostic for acute respiratory injury type 1 and has a maximum inspiratory pressure value of $-20 \mathrm{~cm} \mathrm{H}_{2} \mathrm{O}^{15}$

Patients were excluded if they had any type of acute condition (eg, cardiac arrhythmia) or chronic condition (eg, insufficient congestive heart failure) that could compromise weaning or impede the inspiratory muscle training (neuropathy, myopathy). Tracheostomized patients (in the pretest), patients with neurological problems (cerebral vascular accident, cerebrospinal trauma, spinal medullar trauma), morbidly obese patients, patients receiving medicine that could cause attention disorders, and cases of autoextubation were also excluded. The inclusion and exclusion criteria were evaluated via examination of the patients' medical records and daily physical examination. Informed consent was obtained from the patients' representatives, and the experimental procedures were conducted in accordance with the 1975 Declaration of Helsinki. ${ }^{16}$ This study was submitted to and approved by the Committee of Ethics in Research Involving Human Beings of the Euro-American Network of Human Kinetics (protocol number 005/2007). This trial is registered at ClinicalTrials.gov as NCT00922493.

\section{Procedures}

The evaluation procedures used during pretesting (the first day that the patient initiated ventilation with support pressure) and post-testing (before extubation) were maximum inspiratory pressure and the Tobin index (rate of breath/tidal volume in liters). The outcomes were measured or recorded by physiotherapists in the intensive care unit.

\section{Maximum inspiratory pressure measurements}

Before training, it was necessary to evaluate maximum inspiratory pressure using a vacuum manometer (Critical Care, US, Rio de Janeiro, Brazil). Marini et a ${ }^{17}$ suggested an evaluation method that requires little contribution from the patient. The equipment is attached to the orotracheal tube via 
a connector with an expiratory unidirectional valve, which means there is no resistance in the patient's expiration. Therefore, the individual is obliged to make an inspiratory effort to start the respiratory cycle, at which point the maximum inspiratory pressure is measured. An evaluation duration of 25 seconds produces the best results. ${ }^{18}$ The patients are placed in a dorsal decubitus position with the backrest raised 45 degrees. ${ }^{19}$ The maximum inspiratory pressure cutoff point to be considered for study purposes is $-20 \mathrm{~cm} \mathrm{H}_{2} \mathrm{O} .^{20}$

\section{Measurement of rapid shallow breathing}

A Mark 8 mechanical ventilometer was used (DHD Medical Products, Brooklyn, NY) to calculate the Tobin index. First, the patient was disconnected from the ventilatory prosthesis. Next, the ventilometer was attached to the connecting piece of tube; for one minute, the patient was allowed to breathe spontaneously, ${ }^{15}$ and the current volume and respiratory frequency were measured. For the purposes of the study, the Tobin index cutoff point was $100 \mathrm{ipm} / \mathrm{L} .{ }^{21}$ Inspiratory muscle training was conducted on the first day that the patient initiated spontaneous ventilation and continued until the moment before extubation.

\section{Threshold IMT inspiratory muscle training protocol}

After maximum inspiratory pressure was measured, inspiratory muscle training was initiated. The Threshold-IMT device generates a linear pressure load that produces resistance to inspiration through use of a flow-independent one-way valve. The suggested protocol is as follows: an initial load of $30 \%$ of the maximum inspiratory pressure, increasing daily by $10 \%$, with training for 5 minutes, twice a day, 7 days a week, from the beginning of mechanical ventilation weaning until extubation of the patient. ${ }^{22}$ An essential factor is the use of supplemental oxygen as needed by the patient.

The training was interrupted when respiratory insufficiency and/or hemodynamic instability was observed with any of the following: respiratory frequency $\geq 35 \mathrm{ipm}$ (or higher than $50 \%$ of the initial value); $\mathrm{SpO}_{2}<90 \%$; systolic pressure $>180 \mathrm{mmHg}$ or $<80 \mathrm{mmHg}$ and tachycardia $>140$ beats per minute or $20 \%$ higher than the initial measurement; paradoxical breathing; agitation; depression; hemoptysis; and arrhythmia and/or sweating. ${ }^{23}$ If any of the above clinical signs were observed, the next training session did not involve an additional load. Instead, the training was conducted at the previous level.

The control group followed the protocol of conventional physiotherapy with a reduction in support pressure to $8 \mathrm{~cm}$ $\mathrm{H}_{2} \mathrm{O}$, but without any specific training to strengthen the respiratory musculature. The conventional physiotherapy intervention included passive-assisted to active-assisted mobilization of the limbs, chest compression, decompression traction chest, aspiration of the endotracheal tube, and positioning. Physiotherapy was performed for an average duration of 15 minutes, with the intensity varying according to patient cooperation and vital signs.

For extubation, patients were required to satisfy the following conditions: improvement in the underlying etiology that resulted in respiratory insufficiency, a cough reflex, hemodynamic stability, nonfebrile (body temperature $\leq 38^{\circ} \mathrm{C}$ ), no vasoactive drug use (with the exception of dopamine $5 \mathrm{mg} / \mathrm{kg}$ per minute), stable electrolyte levels (mainly calcium, magnesium, and phosphate) and a normal radiological evaluation (without pneumothorax, congestion, large pleural effusion, or atelectasis). In addition, mechanical ventilation and blood gas analyses were required to fall within the following parameters: $7.30<\mathrm{pH}<7.60$ and $\mathrm{PaCO}_{2}<60 \mathrm{mmHg}$, ${ }^{24}$ inspired fraction of $\mathrm{O}_{2}\left(\mathrm{FiO}_{2}\right) \leq 40 \%$, and $\mathrm{PaO}_{2} / \mathrm{FiO}_{2}$ ratio $\geq 200$. . $^{25,26}$

The patient had to present without signs or parameters of ventilatory discomfort, such as paradoxical breathing, utilization of accessory musculature, respiratory frequency $\geq 35 \mathrm{ipm}$ (or 50\% increase), and sweating.

The protocol for extubation was as follows. The support pressure was reduced to $8 \mathrm{~cm} \mathrm{H}_{2} \mathrm{O}$, ensuring that a minimum volume of $6 \mathrm{~mL} / \mathrm{kg}$ was maintained. Next, a T-tube was utilized for 30 minutes. ${ }^{27}$ Extubation was considered a failure if the patient returned to mechanical ventilation within 48 hours $^{19}$ or required a tracheostomy.

\section{Statistical analysis}

Descriptive statistics, including average and standard deviation, were calculated. The normality of the sample was evaluated with the Shapiro-Wilk test. A kappa test was used to evaluate agreement between the evaluators. A $2 \times 2$ analysis of variance test was used to compare within groups and between groups, and this was followed by a post-hoc Tukey's test to identify any possible differences. A Student's $t$-test was performed to compare permanence times for the noninvasive positive pressure after extubation. A Chisquare distribution analysis was used to evaluate extubation. Sensitivity and specificity were calculated to evaluate the weaning prediction power of the analyzed variables. In addition, the receiver-operating characteristic (ROC) curve was calculated and plotted. The area below the ROC curve for each variable summarizes the performance of each index in predicting weaning. The statistical significance level adopted was $P<0.05$. 


\section{Results}

The baseline characteristics of the two groups are shown in Table 1. Weaning predictor data are presented with the descriptive statistics in Table 2. After the pretest and post-test predictor group results (for maximum inspiratory pressure and Tobin index) were compared, the $2 \times 2$ analysis of variance test indicated significant differences in both variables $(P<0.05)$. As shown in Table 2 , there was a significant and unsatisfactory increase in Tobin index for the control group (95\% confidence interval $[\mathrm{CI}]-4.47$ to $-24.44, P=0.002$ ) and a satisfactory increase in maximum inspiratory pressure in the experimental group (95\% CI 7.09-12.62, $P=0.001)$. The post-test indicated a significant reduction in Tobin index (95\% CI -26.23 to $-6.05, P=0.001)$ and a significant increase in maximum inspiratory pressure (95\% CI 4.67-10.19, $P=0.001)$ when compared with the control group. A kappa test value of 0.68 suggested good inter-rater reliability. Analysis of the extubation results is shown in Figure 1.

The Chi-squared distribution test detected no significant difference in extubation success between the groups $\left(\chi^{2}=1.47 ; P=0.20\right)$, although shorter weaning times were noted in the experimental group (3.64 \pm 1.50 days) compared with the control group (5.36 \pm 1.87 days). However, hospital protocol required reduced ventilation in patients who underwent noninvasive positive pressure after extubation because the procedure is unfavorable for reintubation. Figure 2 shows a comparison of the noninvasive positive pressure time dependence in each group. Note that the experimental group had reduced dependence on noninvasive

Table I Baseline characteristics of patients in the study

\begin{tabular}{lll}
\hline & $\begin{array}{l}\text { Experimental group } \\
(\mathbf{n}=14)\end{array}$ & $\begin{array}{l}\text { Control group } \\
(\mathbf{n}=14)\end{array}$ \\
\hline $\begin{array}{l}\text { Age, years } \\
\text { Gender }\end{array}$ & $82(\mathrm{SD} 4)$ & $8 \mathrm{I}(\mathrm{SD} 6)$ \\
Male & $6(42.86 \%)$ & $7(50.00 \%)$ \\
Female & $8(57.14 \%)$ & $7(50.00 \%)$ \\
Weight, kg & $68(\mathrm{SD} 4.2)$ & $66(\mathrm{SD} 5.9)$ \\
OTT (n) & & \\
7.0 & $2(14.28 \%)$ & $\mathrm{I}(7.14 \%)$ \\
7.5 & $8(57.15 \%)$ & $7(50.00 \%)$ \\
8.0 & $8(57.15 \%)$ & $7(50.00 \%)$ \\
APACHE II, score & $19(\mathrm{SD} 8)$ & $20(\mathrm{SD} 6)$ \\
Causes of ARF & & \\
Postoperative & $2(14.28 \%)$ & $\mathrm{I}(7.14 \%)$ \\
Pneumonia & $8(57.15 \%)$ & $7(50.00 \%)$ \\
Aspiration & $3(21.43 \%)$ & $4(28.58 \%)$ \\
Trauma & $0(0.00 \%)$ & $\mathrm{I}(7.14 \%)$ \\
Sepsis & $\mathrm{I}(7.14 \%)$ & $\mathrm{I}(7.14 \%)$ \\
\hline
\end{tabular}

Abbreviations: OTT, orotracheal tube; APACHE, Acute Physiology and Chronic Health Evaluation; ARF, acute respiratory failure; SD, standard deviation.
Table 2 Results of descriptive statistics and inferential analysis of predictors of weaning

\begin{tabular}{ccc}
\hline & Pretest & Post-test \\
\hline TI & & \\
EG & $73.64 \pm 8.8$ & $79.71 \pm 11.21^{\dagger}$ \\
CG & $81.5 \pm 6.9$ & $95.86 \pm 12.40^{*}$ \\
MIP & & \\
EG & $15.14 \pm 2.57$ & $25.00 \pm 3.90^{*}+$ \\
CG & $15.29 \pm 2.16$ & $17.57 \pm 1.95$ \\
\hline
\end{tabular}

Notes: ${ }^{*} P<0.05$, pretest versus post-test; ${ }^{\dagger} P<0.05$; post-test $E G$ versus pretest. Abbreviations: $\mathrm{TI}$, Tobin index (ipm/L); MIP, maximum inspiratory pressure ( $\mathrm{cm} \mathrm{H}_{2} \mathrm{O}$ ); $\mathrm{CG}$, control group; $\mathrm{EG}$, experimental group.

positive pressure compared with the control group (95\% CI 13.08-18.06, $P=0.0001)$.

An ROC sensitivity and specificity curve was used to evaluate and compare the weaning predictors with data regarding actual successful extubation. The ROC curve for the Tobin index (Figure 3) had an area below the curve of $0.877 \pm 0.06$. The sensitivity and the specificity of the Tobin index was 1.00 and 0.36 , respectively. Sensitivity and specificity as determined by the ROC curve as shown in Figure 4. The curve indicated a sensitivity of 0.86 and a specificity of 0.72 . The area below the curve was $0.845 \pm 0.07$.

\section{Discussion}

Lack of blinding was a limitation of this study. However, informed consent was provided by the relatives of these critically ill patients, so the potential for placebo and Hawthorne effects was reduced. Although we screened 198 patients in the intensive care unit, a large number of these critically ill patients died or were tracheostomized before or after commencement of weaning. This pattern is typical of inspiratory muscle training research in intensive care settings. ${ }^{13,19}$ As a result, another limitation of the study was its sample size. In addition, inclusion of 10 participants allowed an $80 \%$ probability of detecting a difference of $10 \mathrm{~cm} \mathrm{H}_{2} \mathrm{O}$ in maximal inspiratory pressure at a two-sided 5\% significance level. We anticipated that a substantial proportion of these critically ill

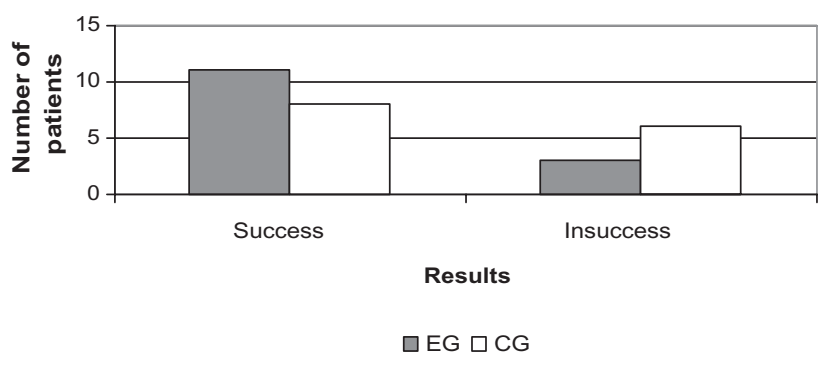

Figure I Evaluation of extubation.

Abbreviations: EG, experimental group; CG, control group. 


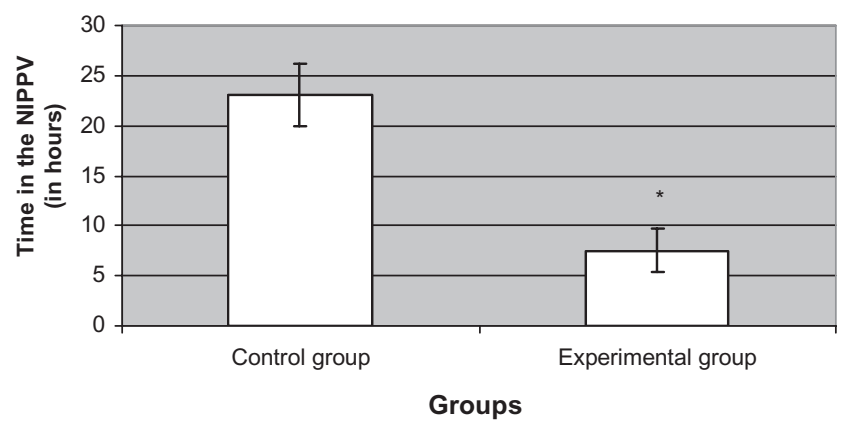

Figure 2 Time on noninvasive positive pressure. Note: $* P<0.05$.

participants would die or receive a tracheostomy, and thus be disqualified from inclusion in our study. Therefore, we increased the sample recruited to 20 to account for possible loss of participants. Therefore, recruitment of 20 participants ensured that the study was adequately powered.

In a study of ventilated patients, Caruso et $\mathrm{al}^{28} \mathrm{did}$ not find that ventilator manipulations provided an effective training stimulus for inspiratory muscles. In contrast, two recent randomized trials demonstrated favorable results with IMT using a removable threshold device. The first study ${ }^{22}$ examined IMT in 28 ventilated patients aged 70 years and older and found that a twice-daily 5 -minute training regimen beginning at an intensity of $30 \%$ of maximum significantly increased the inspiratory muscle strength (mean difference in maximum inspiratory pressure of $7.6 \mathrm{~cm} \mathrm{H}_{2} \mathrm{O}, 95 \%$ CI 5.8-9.4) and a reduction in the weaning period (mean difference of 1.7 days, $95 \%$ CI $0.4-3.0$ ). The second study ${ }^{29}$ investigated

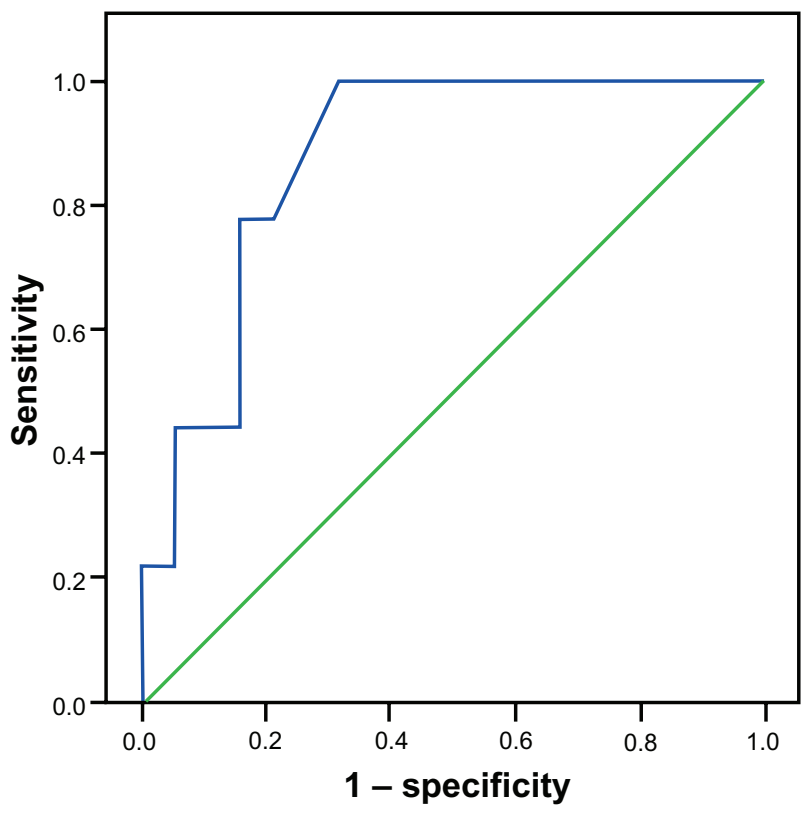

Figure 3 Receiver-operating characteristic curve for Tobin index.

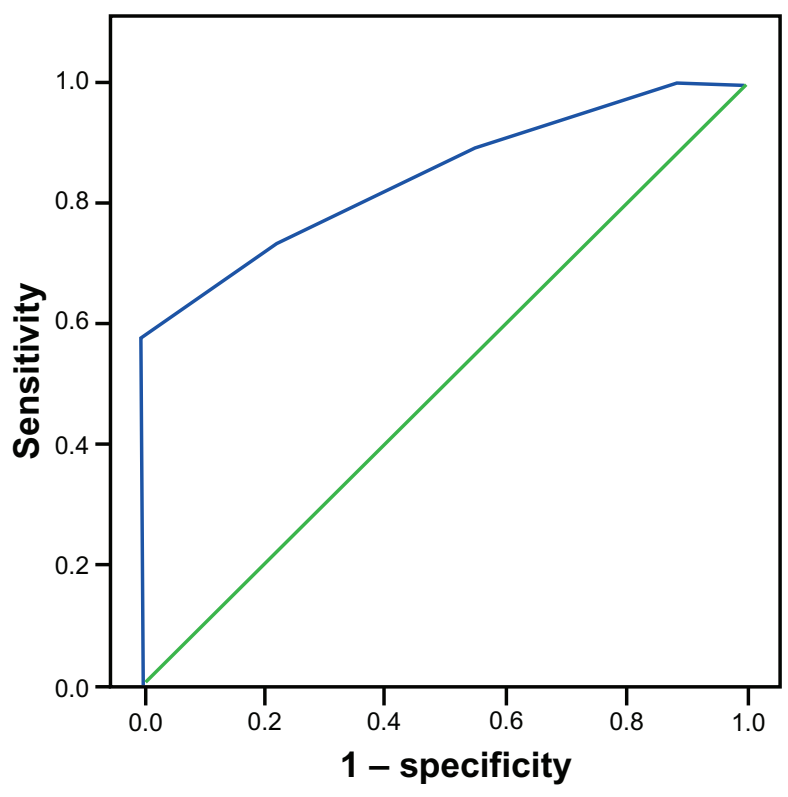

Figure 4 Receiver-operating characteristic curve of maximum inspiratory pressure.

IMT in 69 patients who had failed on conventional weaning methods. These researchers used a high-intensity interval training strategy (ie, four sets of 6-10 breaths) using the highest tolerated resistance. In the IMT group, mean inspiratory muscle strength increased from 44.4-54.1 $\mathrm{cm} \mathrm{H}_{2} \mathrm{O}$, whereas no significant difference was detectable in the sham training group. The training strategies of the two aforementioned studies involved the same training logistics as those in the present study in that they also centered on high-intensity loads. These studies were the focus of a recent systematic review. ${ }^{30}$

Although the results regarding the success of extubation were satisfactory in the present study, the measurements were not significantly different when compared with the control group (Figure 1). However, hospital protocol required that all patients utilize noninvasive positive pressure after extubation, which contributes to airway stabilization and aids in preventing reintubation of patients who might have had extubation issues, as described in the literature ${ }^{31,32}$ (Figure 2).

Limited information is available concerning respiratory muscle training for patients with difficulty weaning, ${ }^{23}$ although some studies describe the contributions of IMT during mechanical ventilation. ${ }^{13,14}$ Chang et al ${ }^{33}$ observed a post-weaning reduction in the inspiratory muscle strength of patients who had undergone a lengthy period of mechanical ventilation. These results are in agreement with those for the control group, as shown in Table 2.

Figure 3 shows that the Tobin index has high sensitivity and low specificity, which is in agreement with a study by Yang and Tobin, ${ }^{15}$ who observed that the Tobin index has 
a sensitivity of 0.97 and a specificity of 0.64 . Utilizing a cutoff of $-17 \mathrm{~cm} \mathrm{H}_{2} \mathrm{O}$ for maximum inspiratory pressure, Conti et $\mathrm{al}^{23}$ found a sensitivity of up to 0.95 and a specificity of 0.42 . Although the cutoff value is not identical to the one used in our study $\left(-20 \mathrm{~cm} \mathrm{H}_{2} \mathrm{O}\right)$, the sensitivity and specificity values for maximum inspiratory pressure showed the same trend as those shown in Figure 4 (high sensitivity and low specificity). However, as previously mentioned, the low specificity values also justify use of noninvasive positive pressure ventilation.

\section{Conclusion}

From our study sample, it can be inferred that inspiratory muscle training contributed to a significant increase in maximum inspiratory pressure (both within groups and between groups) and a reduction in Tobin index (within groups). These variables are considered good weaning predictors, and their related ROC curves indicate that IMT was associated with reduction in noninvasive positive pressure time in the experimental group.

\section{Disclosure}

The authors report no conflicts of interest in this work.

\section{References}

1. Janssens JP, Pache JC, Nicod LP. Physiological changes in respiratory function associated with ageing. Eur Respir J. 1999;13(1):197-205.

2. Ambrosino N. Weaning and respiratory muscle dysfunction: the eggchicken dilemma. Chest. 2005;128(2):481-483.

3. Alfieri FM, Riberto M, Gatz LS, Ribeiro CP, Lopes JA, Battistella LR. Comparison of multisensory and strength training for postural control in the elderly. Clin Interv Aging. 2012;7:119-125.

4. Vinsnes AG, Helbostad JL, Nyronning S, Harkless GE, Granbo R, Seim A. Effect of physical training on urinary incontinence: a randomized parallel group trial in nursing homes. Clin Interv Aging. 2012;7:45-50.

5. Vassilakopoulos T, Petrof BJ. Ventilator-induced diaphragmatic dysfunction. Am J Respir Crit Care Med. 2004;169(3):336-341.

6. Meade M, Guyatt G, Cook D, et al. Predicting success in weaning from mechanical ventilation. Chest. 2001;120(Suppl 6):400S-424S.

7. Robriquet L, Georges H, Leroy O, Devos P, D'Escrivan T, Guery B. Predictors of extubation failure in patients with chronic obstructive pulmonary disease. J Crit Care. 2006;21(2):185-190.

8. Bruton A. A pilot study to investigate any relationship between sustained maximal inspiratory pressure and extubation outcome. Heart Lung. 2002;31(2):141-149.

9. Vallverdu I, Calaf N, Subirana M, Net A, Benito S, Mancebo J. Clinical characteristics, respiratory functional parameters, and outcome of a two-hour T-piece trial in patients weaning from mechanical ventilation. Am J Respir Crit Care Med. 1998;158(6):1855-1862.

10. Goldstone J. The pulmonary physician in critical care. 10: difficult weaning. Thorax. 2002;57(11):986-991.

11. Volianitis S, McConnell AK, Koutedakis Y, Jones DA. Specific respiratory warm-up improves rowing performance and exertional dyspnea. Med Sci Sports Exerc. 2001;33(7):1189-1193.
12. Appendini L, Purro A, Patessio A, et al. Partitioning of inspiratory muscle workload and pressure assistance in ventilator-dependent COPD patients. Am J Respir Crit Care Med. 1996;154(5):1301-1309.

13. Chang AT, Boots RJ, Brown MG, Paratz J, Hodges PW. Reduced inspiratory muscle endurance following successful weaning from prolonged mechanical ventilation. Chest. 2005;128(2):553-559.

14. Del Rosario N, Sassoon CS, Chetty KG, Gruer SE, Mahutte CK. Breathing pattern during acute respiratory failure and recovery. Eur Respir J. 1997;10(11):2560-2565.

15. Yang KL, Tobin MJ. A prospective study of indexes predicting the outcome of trials of weaning from mechanical ventilation. $N$ Engl $J$ Med. 1991;324(21):1445-1450.

16. World Medical Organization. Declaration of Helsinki. BMJ. 1996;313: $1448-1449$.

17. Marini JJ, Smith TC, Lamb V. Estimation of inspiratory muscle strength in mechanically ventilated patients: The measurement of maximal inspiratory pressure. J Crit Care. 1986;1(1):32-38.

18. Caruso P, Friedrich C, Denari SD, Ruiz SA, Deheinzelin D. The unidirectional valve is the best method to determine maximal inspiratory pressure during weaning. Chest. 1999;115(4):1096-1101.

19. Sprague SS, Hopkins PD. Use of inspiratory strength training to wean six patients who were ventilator-dependent. Phys Ther. 2003;83(2):171-181.

20. Afessa B, Hogans L, Murphy R. Predicting 3-day and 7-day outcomes of weaning from mechanical ventilation. Chest. 1999;116(2):456-461.

21. Epstein SK, Ciubotaru RL. Influence of gender and endotracheal tube size on preextubation breathing pattern. Am J Respir Crit Care Med. 1996;154(6):1647-1652.

22. Cader SA, Vale RG, Castro JC, et al. Inspiratory muscle training improves maximal inspiratory pressure and may assist weaning in older intubated patients: a randomised trial. $J$ Physiother. 2010;56(3):171-177.

23. Conti G, Montini L, Pennisi MA, et al. A prospective, blinded evaluation of indexes proposed to predict weaning from mechanical ventilation. Intensive Care Med. 2004;30(5):830-836.

24. Venkataraman ST, Khan N, Brown A. Validation of predictors of extubation success and failure in mechanically ventilated infants and children. Crit Care Med. 2000;28(8):2991-2996.

25. Martinez A, Seymour C, Nam M. Minute ventilation recovery time: a predictor of extubation outcome. Chest. 2003;123(4):1214-1221.

26. Frutos-Vivar F, Ferguson ND, Esteban A, et al. Risk factors for extubation failure in patients following a successful spontaneous breathing trial. Chest. 2006;130(6):1664-1671.

27. Boles JM, Bion J, Connors A, et al. Weaning from mechanical ventilation. Eur Respir J. 2007;29(5):1033-1056.

28. Caruso P, Denari SD, Ruiz SA, et al. Inspiratory muscle training is ineffective in mechanically ventilated critically ill patients. Clinics. 2005;60(6):479-484.

29. Martin AD, Smith BK, Davenport PD, et al. Inspiratory muscle strength training improves weaning outcome in failure to wean patients: a randomized trial. Crit Care. 2011;15(2):R84.

30. Moodie LH, Reeve JC, Vermeulen N, Elkins MR. Inspiratory muscle training to facilitate weaning from mechanical ventilation: protocol for a systematic review. BMC Res Notes. 2011;4:283.

31. Matte P, Jacquet L, Van Dyck M, Goenen M. Effects of conventional physiotherapy, continuous positive airway pressure and non-invasive ventilatory support with bilevel positive airway pressure after coronary artery bypass grafting. Acta Anaesthesiol Scand. 2000;44(1):75-81.

32. Hilbert G, Gruson D, Vargas F, et al. Noninvasive ventilation in immunosuppressed patients with pulmonary infiltrates, fever, and acute respiratory failure. $N$ Engl J Med. 2001;344(7):481-487.

33. Chang AT, Boots RJ, Henderson R, Paratz JD, Hodges PW. Case report: inspiratory muscle training in chronic critically ill patients - a report of two cases. Physiother Res Int. 2005;10(4):222-226. 
Clinical Interventions in Aging

\section{Publish your work in this journal}

Clinical Interventions in Aging is an international, peer-reviewed journal focusing on evidence-based reports on the value or lack thereof of treatments intended to prevent or delay the onset of maladaptive correlates of aging in human beings. This journal is indexed on PubMed Central, MedLine, the American Chemical Society's 'Chemical Abstracts Ser-

vice' (CAS), Scopus and the Elsevier Bibliographic databases. The manuscript management system is completely online and includes a very quick and fair peer-review system, which is all easy to use. Visit $\mathrm{http}: / /$ www.dovepress.com/testimonials.php to read real quotes from published authors. 